\title{
Study on BIM Modeling Method of Bridge Railway Integration Based on Revit and Civil3D
}

\author{
Lv Siqing ${ }^{1, a}$ \\ ${ }^{1}$ School of Civil Engineering,Sichuan Agricultural University,Dujiangyan,Sichuan,Jianshe Road \\ No.288,China \\ ats_650@sina.com
}

Keywords: BIM model, frame bridge, rail structure, parametric family model, model registration, rendering and roaming.

Abstract. To use BIM technology in the whole life cycle of railway structures, this paper builds all parts family models of frame bridge by Autodesk Revit including body and foundation of frame bridge, wing wall and its foundation, leak bed and appurtenant works on the top of bridge. Building track model by using secondary development of Civil 3D and importing it into Revit. Assembling BIM parametric model of the intact frame bridge based on each part's control parameters, geometric constraint conditions and association relationship. Then introducing the frame bridge model into the software platform of Lumion to realize its three-dimensional dynamic roaming show. This paper provides new method and theory for $3 \mathrm{~d}$ visualization show of this bridge structure.

\section{Introduction}

With the unprecedented development of railway construction, it is imperative to increase the production efficiency of relevant professionals such as railway planning, design, construction and maintenance.In particular, there is an urgent need to improve the production efficiency in the planning and design stage of railway line[1].

In recent years,the rapid development of BIM technology has injected new vitality into the entire infrastructure construction field and has been hailed as a revolutionary force in the transformation of the construction industry[2].For example,when Disney castle was built in Shanghai,the model was established by Revit in the initial stage of design and that changed the traditional CAD drawing method.It can realize automatic generation of construction drawings and collision detection,pipeline layout at the later stage[3]. Each domestic design institutes have gradually begun to use BIM technology for design in urban rial transit,bridges,and long-to-large tunnels[4].The bridge design transforms from traditional isolated and stationary 2D design to 3D collaborative design, it realizes the overall design optimization of bridge and brings great success to the subsequent data mining,the optimization flow is showed in figure.1.Practice shows that BIM technology is more technologically innovative and can simplify 2D plane design,increase the level of automation of computer-aided design and significantly shorten design cycle[5]. However, independent BIM design of bridge can no longer meet the needs of project.The integration of BIM includes bridges, tunnels, embankments, tracks and terrain is the trend of BIM development in the railway industry.This paper conducts a preliminary exploration of BIM model nesting for bridge and track. 


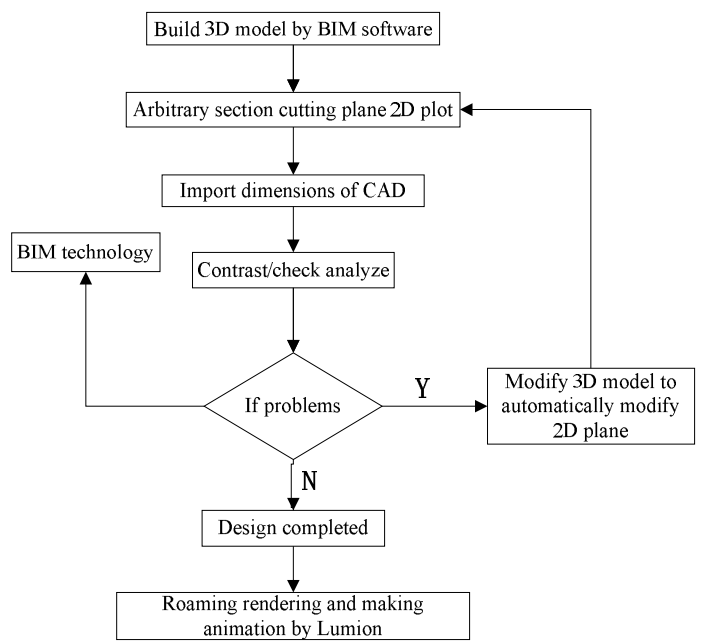

Fig.1 Optimization process of bridge design based on BIM technology

\section{Modeling environment}

In consideration of structural characteristics and advantages, disadvantages in each software platform of frame bridge BIM model, Revit is chosen as the modeling platform[6]. Revit's family is its core feature and basis for designing all components which includes three-dimensional information and attribute information.The family can realize assemblage of same type components.Modifying parameters can get different types of components in the family.When designing,the family library is used to call different types of families to create family instances and the whole building is assembled[7].

\section{Project profile}

The bridge is a $4-6 \mathrm{~m}$ frame bridge with a length of $26.24 \mathrm{~m}$.It is constructed of on-site concrete.The frame body is made of C35 concrete,frame body foundation, wing wall body and wing wall foundation are made of C30 concrete.Based on the BIM technology, it mainly discusses BIM modeling method for the main body,superstructure,wing wall and its foundation.Establish the corresponding family library in Revit software platform and that can realize rapid construction of BIM model of the frame bridge. The model is built as shown in figure.2:

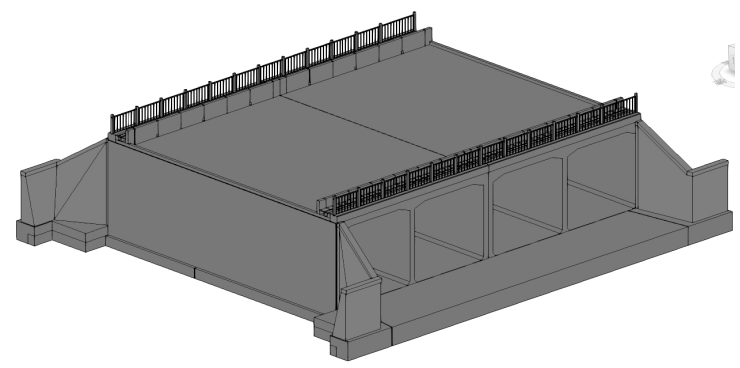

Fig.2 3 Dimensions model of frame bridge

\section{BIM modeling method for body and foundation of frame bridge}

According to different modeling objects in the frame bridge, set corresponding control parameters, geometric constrains and associated relationships. Different reference planes adopt corresponding modeling methods which mainly include stretching, setting out, fusion, slotting, 
punching, emptying and so on. Establish family model of each component structure in order to achieve real-time changes and overall unity of engineering design information.By modifying its control parameters to realize automatic modification of the overall model,load each part of family model into the frame bridge model project and use dimensions,position correlation to assemble and complete three-dimensional modeling of the entire frame bridge(Figure.2).

\section{BIM model establishment of frame bridge body}

The method for building frame body family model is relatively simple, and it can be completed only by using stretching and hollow stretching commands in the metric conventional model. When modeling,it should pay attention to the settings of reference elevation and planes to prevent failing of nesting among families.Family nesting of frame bridge body and foundation is shown in figure.3:

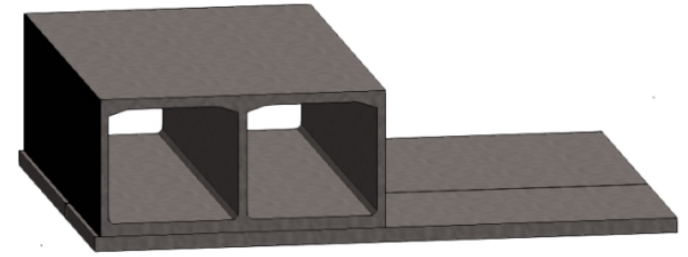

Fig.3 Family nesting of foundation and body

In the process of establishing a family model,parametric settings such as lengths, widths, heights, angles and materials should be synchronized. The material information added for the model is not the text in traditional model,but the 'real material' with material properties,and the property values can be modified.

\section{BIM model establishment of wing wall,wing wall foundation and leak bed}

Since the Revit software is mainly for architectural profession,modeling for special structural parts in bridge has limitations. The establishment of wing wall family model can not be realized by family-metric conventional model. Select concept volume-metric to create,draw the bottom outlines,pick up key nodes in 3D view and draw a spatial spline curve,select solid shape under the create shape command.Wing wall model is shown in figure.4:

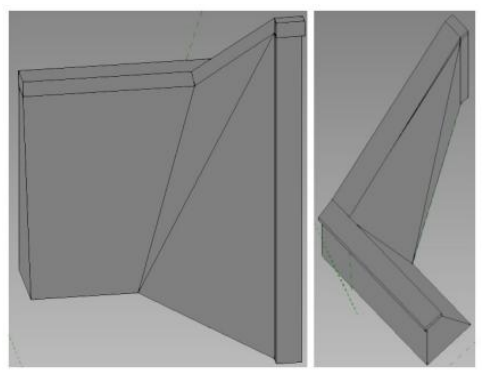

Fig.4 Family model of wing wall

The wing wall foundation and leak bed family model are established by using stretching and fusion commands, settlement joints are cut by hollow stretching.As shown in figure.5,the upper layer is C30 concrete and lower layer is gravel cushion.

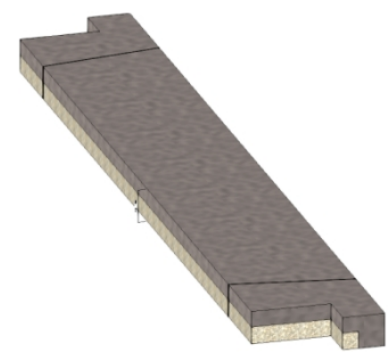

Fig.5 Family model of wing wall foundation and leak bed 


\section{BIM model establishment of frame bridge's top appurtenant works}

The top of frame body mainly includes capstones,cable troughs,cable troughs covers and bump walls.In the modeling process of each part,the position relationship between them is mainly considered. Therefore, it is necessary to associate each size label and set multiple reference planes, simultaneously use of family nesting to complete the top overall BIM modeling.Because the capture function of Revit is inaccurate,mirroring,rotation and array commands are used in the modeling process to simplify the modeling process and improve work efficiency. Model nesting of cover family and the top overall is shown in figure.6.

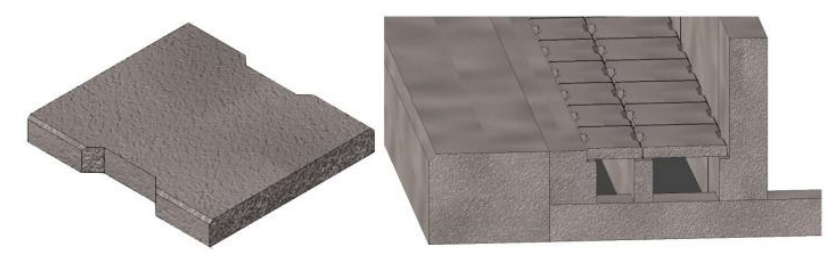

Fig.6 Nesting of parametric cover family model and frame bridge's top appurtenant works Select the rail command to draw path,rail model building is shown in figure 7:

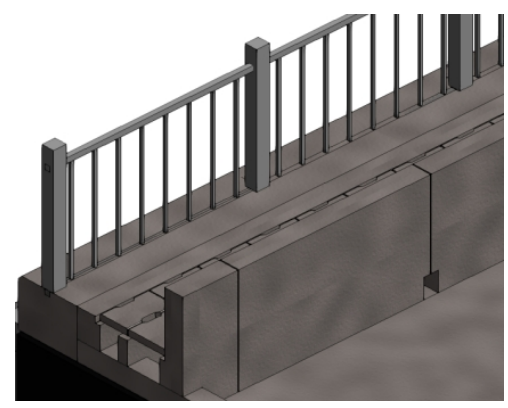

Fig.7 Handrail model

\section{Modeling method of track structure,roadbed and terrain}

Rail takes the standard 60 cross-section profile as an example,take the middle line of section as boundary and take the geometric parameter coordinates of the section as an example,calculate the coordinates of each control point of the rail cross section in plane coordinate system. Through Visual Basic editor to do the secondary development of Civil 3D so that it can automatically generate the rail model.Sleepers,fasteners and track beds are complicated in structure and their dimensions are basically fixed.Use the method of creating a three-dimensional library,write programs, drawing process is automatically completed inside of computer.A window can be set to facilitate the designers to call component models. Window shown in figure. 8 is programming process of macros interface window in Civil 3D.

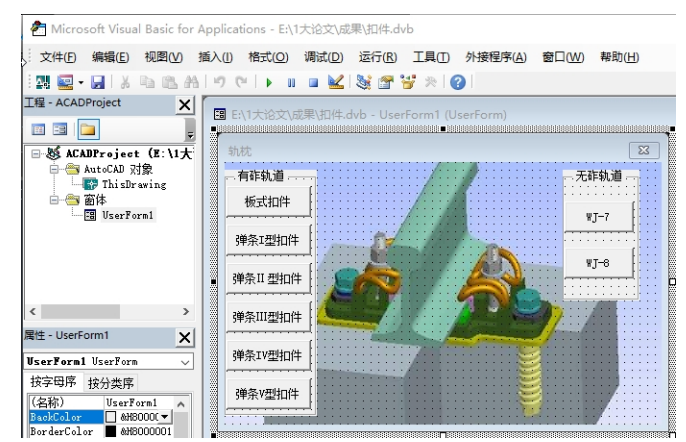

Fig.8 Schematic diagram of fastener drawing window 
Programming as follows:

ThisDrawing.ModelSpace.AddCircel ptl,radius

ThisDrawing.ModelSpace.AddCircel pt2,radius

UserForml.Hide

Dim objcyliner As Acad3DSoild

Dim tdb As Acad3DSoild

Dim objList ( 0 to 1) As AcadEntity

Dim obRegion As Variant

Set objlist1 (0) =ThisDrawing.ModelSpace.AddCircel (pt1,radius)

Set objlist1 (1) = ThisDrawing.ModelSpace.AddCircel (pt2,radius)

objList (0) .Delete

objList (1).Delete

Set $\mathrm{tdb}=$ ThisDrawing.ModelSpace.AddExtrudedSolid（objregion（0）,-h,0）

Build fastener,sleeper,elastic bar,circular boss as figure.9.

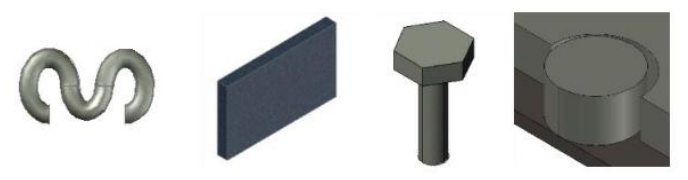

Fig.9 Schematic diagram of component models

After selecting fasteners, track beds,rails and other structural elements, the three-dimensional model of each parts is automatically established and the entire model is completed in accordance with assembling process. The result is shown in figure. 10 .

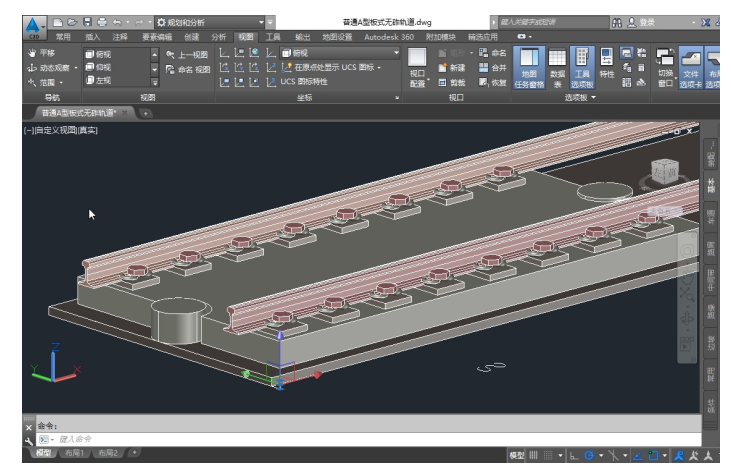

Fig.10 Building schematic diagram of A type slab ballastless track

The track model is exported to Dwg format and linked to the frame bridge model in Revit.The volume and site commands are used to import the coordinates of terrain points, and coordinates of nesting points are captured according to the spatial position relationship of different structures.The nesting model of track,roadbed,frame bridge and terrain is shown in figure. 11.

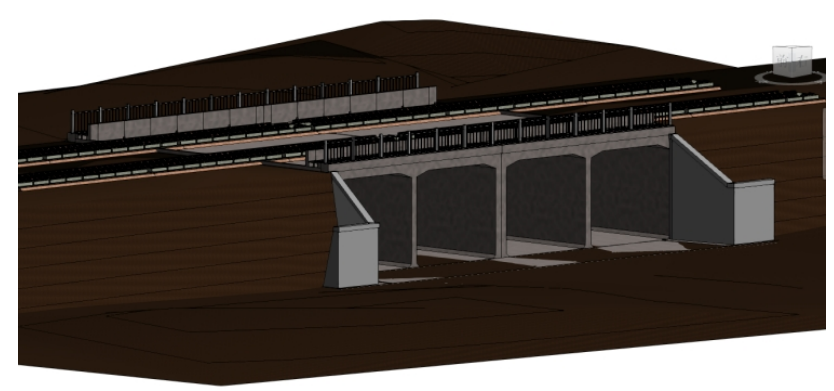

Fig.11 Nesting schematic diagram of railway, bed, terrain and frame bridge 


\section{Construction drawing export and calculation check}

It can sectioning arbitrary surface in Revit according to actual needs of engineering design,the mutual relationship among building components can be displayed intuitively and quickly,three dimensional axonometric drawing of frame bridge can be plotted form various angles and it makes problems which are difficult to think in two dimensional design be clear at a glance[8].

BIM model has obvious advantages in project statistics, collision check and $3 \mathrm{~d}$ visualization show[9]. After the model is created and plotted, in Revit list it can realize project statistics which includes different materials, families, components. Then do statistics of actual project measurement and calculate the statistics result. Compare the result with calculation list of designers', the accuracy of calculation is improved.

\section{Three-dimensional roaming of BIM model}

Lumion is a real-time 3D visualization tool for creating animation and static-frame works. The software has rich 3D materials and scene models, but it can not create 3D models.It can only be used to add materials, attach images, render and make dynamic roaming.Lumion software can provide a visualization platform for project participants to directly and vividly visualize the whole view of project.Specific steps are as follows:

(1)Using Lumion software to perform 3D roaming of frame bridge,first install'Revit to Lumion bridge' conversion plug-in and keep Lumion running;

(2)Export Revit BIM model of frame bridge in DAE format;

(3) Select scene which Lumion contains,import the 3D solid model.The elevation of model datum is same as default elevation of Lumion scene datum. Therefore,select the appropriate site to place the frame bridge model;

(4) Select an appropriate roaming route can reflect position,structure, details, etc of the frame bridge from third perspective,full-scale and multiple angle view[10].(Figure.12)

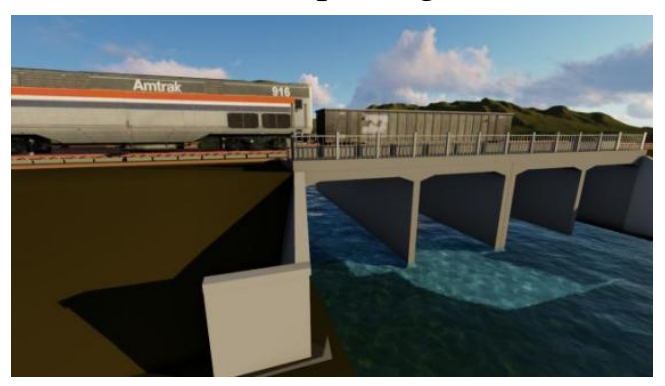

Fig.12 3D roaming of frame bridge

\section{Conclusions}

Through creation of a complete and sophisticated 3D integration model of frame bridge and railway, use of BIM technology allow subsequent comparison of schemes, construction process simulation,operation and maintenance management. The establishment of family library can meet centralized storage and management of components. Frame bridge is used frequently in engineering projects, the BIM parametric modeling method of frame bridge can quickly modeling for frame bridge of different sizes.It has high versatility that is not available in traditional modeling method. This article creates family models of each part in frame bridge, then ensure every family model's three dimensional position in detail from reference plane and elevation.Assemble each family 
component to get the whole BIM model of frame bridge. Combined with 3D roaming software,the overall and detailed structure of frame bridge can be intuitively and vividly displayed. This is incomparable by the traditional plane design. Due to the limitations of Revit itself, it is necessary to pay attention in the BIM parametric modeling process:

(1)Use the families provided in Revit to select different family templates based on the families' own characteristics.

(2) Set different control parameters, constrains, relationships, reference planes and elevations for different modeling objects.

(3) As the BIM concept runs through entire life cycle of project, it must involve multiple software platforms that interact and work together. Therefore, creation of BIM parametric model needs to consider the requirements of each stage in advance.

\section{References}

[1] Miao Yu: Application of BIM in railway design.Transport Construction \& Management. Vol. 201-202(2014), p.20

[2] Jun Qin:BIM application at architectural design stage.Architecture Technique. Vol. 160-163(2011), p. 1

[3] Yaodong Zhang, Min Yang,Haining Gong:Analysis of application of BIM technology in Shanghai Disney magical fairy tale castle. Water \& Wastewater Engineering. Vol. 62-66(2014),p.7

[4] Yongjie Pan:BIM based bridge construction and operation maintenance integration platform.Railway Computer Application.Vol.39-42(2016),p.5

[5] Feng Qian:Bridge engineering BIM technology application research.Railway Standard Design.Vol.51-52(2015),p.12

[6] Sheng Wang:Analysis of BIM and its tools--The choice of BIM software.Intelligent City.Vol.289-290+29(2016),p.11

[7] Kun Cao:Research parametric modeling method of structure model of BIM on railway line.Southwest Jiaotong University(2016)

[8] Jianjun Zhang,Xiao Yang,Hongshen Ma.et al:Study on bridge BIM design method based on Autodesk Revit.China Municipal Engineering.Vol.94-97(2016),p.4

[9] Dengfeng Yang:Application of BIM technology in road and bridge design stage.China Science and Technology Information. Vol.43-46(2017),p.8

[10] Yibei Zhu,Yaodong Cheng,Lizhao Xie:BIM modeling of PC continuous box-girder bridge stiffened by steel truss.Railway Standard Design.Vol.88-92(2017),p.4 